\title{
Lithium in Giant Stars
}

\author{
Ramiro de la Reza \\ Observatório Nacional, Rio de Janeiro, 20921-400 Brazil
}

\begin{abstract}
Lithium continues to be a surprising element and more than ever the study of its creation, destruction and distribution is giving us tools to understand, not only the chemical evolution of this element, but also the nature of mass loss of evolved giants and nucleosynthesis of other elements in cases of very low-metal giants. It also helps to set constraints for cosmological models. The presence of very strong lithium lines in some giant stars of different spectral types and stages of evolution has been considered up to the end of the nineties as a puzzle. To solve this problem, non standard evolutionary mechanisms must be invoked. We review here all the mechanisms presented in the literature and which are divided into internal and external processes of lithium enrichment. We will also discuss the observational tests which are being performed in order to discard (or not) some of them. In any case, the more realistic values of the lithium abundances in giants are, as we will see, the main test of these proposed scenarios. Because of this importance we discuss here the state of art of the Non-LTE determinations of lithium abundances in strong lithium giants. Evolved giants, with lithium abundances larger than that of the interstellar medium and with their important mass losses can be considered the most realistic sources of lithium in the Galaxy. We believe that a complete physical picture of this problem will give a powerful tool to understand the chemical evolution of a large part of all light elements.
\end{abstract}

\section{Introduction}

Three discoveries, realized in very different years, 1940, 1982 and 1989 put in evidence the existence of very strong lithium giants. Mac Kellar (1940) detected a very strong $\mathrm{Li}$ I resonance line in the spectrum of WZ Cas, a cool carbon star located in the Asymptotic Giant Branch (AGB). Much later, Wallerstein \& Sneden (1982) detected the first Li strong K giant (HD 112127), being a first ascending red giant branch star (RGB). In 1989, Smith \& Lambert (1989) detected several Li strong S and MS type AGB giants in the Magellanic Clouds. Today we know several of these stars, nevertheless, their number in respect to the Li-poor giants is only known approximately. It depends on the kind of giant stars and on the definition of the minimum abundance defining a Li-rich star. The values oscillate between $2 \%$ and $8 \%$ according to different authors. As we will see, this proportion appears to increase to $60 \%$ among the fast rotating giants. In any case, the presence of these very strong and also moderately strong $\mathrm{Li}$ lines is opposed to what is expected from the first dredge-up standard 
evolutionary theory where, on the contrary, only Li-poor giants are expected to exist. This situation has received names such as "the Lithium problem" "or "the 'puzzle of the Lithium" " in the literature.

Even today, 60 and 18 years respectively after the discoveries of Li-strong giants among the carbon and $\mathrm{K}$ type stars, we have to decide between the internal and external scenarios for the Li enrichment in giants. The internal mechanisms are all based on the ${ }^{7} \mathrm{Be}$ mechanism (Cameron 1955; Cameron \& Fowler 1971), whereas the external ones are based on the direct injection of ${ }^{7} \mathrm{Li}$ into the atmospheres of the giant produced by hot nova companions or by engulfing of planets or brown dwarf companions by the giant stars.

In the following, we will discuss the different scenarios that appeared in the literature. For each model the present or future observational tests that could confirm or discard these models will be indicated. Afterwards, we will present the state of art of the Non-LTE methods employed to determine the abundances of $\mathrm{Li}$ in giants. At the end, we will present some conclusions and perspectives concerning this problem.

\section{Internal Lithium Enrichment Mechanisms}

\subsection{The Internal Mixing and Convective Scenarios}

These are based in the efficiency of the ${ }^{7} \mathrm{Be}$ mechanism, in which ${ }^{7} \mathrm{Be}$ is produced in the hot internal layers corresponding to the H-burning zone and which are necessary to produce the reaction ${ }^{3} \mathrm{He}(\alpha, \gamma){ }^{7} \mathrm{Be}$. For giants with masses between 3.5 and $6 M_{\odot}$ this reaction is produced very near the internal base of the convective envelope, and in this way, fresh ${ }^{7} \mathrm{Be}$ can be rapidly transported to the external layers where it can be transformed into ${ }^{7} \mathrm{Li}$ by ${ }^{7} \mathrm{Be}\left(e^{-}, \nu\right)^{7} \mathrm{Li}$ before being destroyed by reactions as ${ }^{7} \operatorname{Li}(p, \alpha)^{4} \mathrm{He}$ or at hotter temperatures by ${ }^{7} \mathrm{Be}(p, \alpha){ }^{4} \mathrm{He}$. These are the main ingredients of the scenario called 'Hot Bottom Burning (HBB) which have been developed in detail by Sackmann \& Boothroyd (1992), and Mazzitelli, D' 'Antona \& Ventura (1999). For giants with masses equal or larger than $7-8 M_{\odot}$ the ${ }^{7} \mathrm{Be}$ mechanism is no more in action. This is because the internal temperatures in this case are hot enough to destroy all the ${ }^{7} \mathrm{Be}$ at the base of the convection zone. The HBB reproduces very well the existence of Li-rich AGB giants of types S and MS discovered by Smith \& Lambert (1989) in the Magellanic Clouds.

Now, let us consider the situation of low mass giants with masses $<2.5 M_{\odot}$. Because the temperatures at the base of the convective layer are not hot enough to produce ${ }^{7} \mathrm{Be}$, an internal mixing mechanism has to be invoked in order to connect the base of the convective layer to the H-burning zone. For this purpose an ad-hoc conveyor belt has been introduced by Sackmann \& Boothroyd (1999) which is able to produce ${ }^{7} \mathrm{Li}$ surface enrichments as large as $\log \varepsilon(\mathrm{Li})=4.2$ for low-metal giants with $[\mathrm{Fe} / \mathrm{H}] \sim-2.3$ due to the hotter internal temperatures attained in these giants. This mechanism is called 'Cool Bottom Processing' (CBP) and has its efficiency increased due to the existence of internal excesses of ${ }^{3} \mathrm{He}$ in only this range of stellar masses.

Because of the efficiency of the CBP in very metal-poor giants all the ${ }^{3} \mathrm{He}$ can be consumed in the RGB and in this way no Li-rich AGB giants are expected to exist for this extreme case (Sackmann \& Boothroyd 1999). Details of these 
mechanisms can also be found in Sackmann \& Boothroyd in these proceedings. Which mechanism is acting in the intermediate range of masses between 2.5 and $3.5 M_{\odot}$ ? Probably the conveyor belt, reducing its importance and passing smoothly from the CBP to the HBB case.

Observational tests of this model can be made by observing the abundances of the primordial ${ }^{9} \mathrm{Be}$ and ${ }^{10,11} \mathrm{~B}$ abundances in these Li-rich giants. CBP predicts that these elements, having existed in the atmospheres since the creation of these stars, will vanish rapidly by the action of the deep mixing process. In fact, the first ${ }^{9} \mathrm{Be}$ observations in Li-rich giants have shown that this is the case (Castilho et al. 1999, see also Castilho in these proceedings). Because ${ }^{6} \mathrm{Li}$ is the most fragile of the primordial elements we expect that a large depletion of this isotope will be produced in a continued action of the CBP.

\subsection{The Internal Rotation Induced Mechanisms}

Low-mass giants are in general low rotation giants with $v \sin i \sim 2 \mathrm{~km} \mathrm{~s}^{-1}$, however, the existence of some giants with large rotational velocities have suggested in the past that in some way the external transport of momentum of a high rotation core could be the cause of relatively fast external rotations and even of mass loss. Fekel (1988) first suggested that ${ }^{7} \mathrm{Li}$ could be the clue to understand this process. Later Fekel \& Balachandran (1993) developed the idea in which ${ }^{7} \mathrm{Be}$ is transported to the exterior, helped by this transfer of momentum, but not exists a one to one relation between rotation and $\mathrm{Li}$ abundances, because ${ }^{7} \mathrm{Li}$ enrichment and rotation decay times are not necessary the same. More recently, Drake et al. (2000) have found a clear correlation existing between very fast rotation $\left(v \sin i \geq 8 \mathrm{~km}^{-1}\right)$ and high and very high $\mathrm{Li}$ abundances. This correlation appears, however, only when the giants present clear evidences of mass loss through the presence of large excesses of far infrared (FIR) radiation and also through quite peculiar asymmetric $\mathrm{H} \alpha$ absorption lines. This is the case of giants HD 233517, HD 219025 and PDS 365. Interestingly, Drake et al. (2000) have also shown that considering moderately Li-strong giants together with the $\mathrm{Li}$-strong ones, the proportion of Li-rich objects increases to $60 \%$ ! among the high rotation stars. Somehow, rotation induces the reduction of Li depletion. This rotation induced mechanism has to explain the existence of very Li-rich $\mathrm{K}$ giants with very low rotation velocities $\left(v \sin i \sim 1 \mathrm{~km} \mathrm{~s}^{-1}\right)$ as is the case of HD 19745 (de la Reza \& da Silva 1995).

\subsection{The Prompt ${ }^{7}$ Li Enrichment - Mass Loss Scenario}

The discovery of the fact that a very large part of the low-mass Li-rich giants are the optical counterparts of IRAS point sources (Gregório-Hetem et al. 1992; Gregório-Hetem, Castilho, \& Barbuy 1993) led to a scenario in which all RGB stars pass by a short interval of time $\left(10^{3-}-10^{5} \mathrm{yrs}\right)$ during which these giants are Li-rich (de la Reza, Drake, \& da Silva 1996; de la Reza et al. 1997; see also de la Reza et al. in these proceedings). This 'Li cycle “is produced by a sudden ${ }^{7} \mathrm{Li}$ enrichment of internal origin producing the formation of a circumstellar shell (CS). When this internal rapid mechanism ceases, the CS is detached from the star, transporting the new produced ${ }^{7} \mathrm{Li}$ into the interstellar matter contributing this way to the Galaxy enrichment with this element. The fresh ${ }^{7} \mathrm{Li}$ that remains in the photosphere of the stars is gradually depleted by convection in 
times of $10^{3}$ to $10^{5}$ yrs for expansion velocities of the CS of less than $5 \mathrm{~km} \mathrm{~s}^{-1}$ and episodic mass losses of the order of $\sim 5 \times 10^{-8} M_{\odot} / \mathrm{yr}$. Being ${ }^{3} \mathrm{He}$ the 'fuel ${ }^{2}$ by which the ${ }^{7} \mathrm{Be}$ mechanism came into action, the $\mathrm{Li}$ cycle can be repeated depending on the abundance of the internal remaining ${ }^{3} \mathrm{He}$. The repeated action can produce eventual multiple CS. This Li cycle is represented in a color-color diagram containing the fluxes at 12,25 and 60 microns, by closed curves representing the CS expansion and passing over the observed distribution of $\mathrm{K}$ giants in this diagram. Whereas the main bulk of $\mathrm{K}$ giants, which are Li-poor, is concentrated in a region of this diagram presenting no CS, the Li-rich and Li-poor giants with CS having FIR excesses are distributed in various regions of the diagram. Because the CS ejection and Li depletion in stars are time synchronized, the depletion times can be estimated by the position of the Li-poor $\mathrm{K}$ giants in this diagram. An alternative model of this scenario, in which the velocity of the CS depends on the mass of the CS, is presented by Torres et al. in these proceedings.

Another way to examine the $\mathrm{Li}$ enrichment in this scenario in the RGB stage, consists in relating it to the luminosity bump that appears in this evolutionary stage. This bump corresponds to the evolutionary stage when the hydrogen-burning shell erases the chemical discontinuity left behind by the first dredge-- up at the moment when the convective layer was at its maximum extent or deepest penetration. Because the CBP is expected to start at this stage until the tip of the RGB is attained, we expect (if the CBP is responsible for the ${ }^{7} \mathrm{Li}$ enrichment) to observe Li-rich giants at luminosities equal or higher than the RGB bump. We verified that this is effectively the case, using all $\mathrm{Li} \mathrm{K}$ giants for which we have Hipparcos distances and using the bumps proposed by Charbonnel (1994) for giants with masses between 1.0 to $1.5 M_{\odot}$ (see also Charbonnel et al. in this proceedings).

What is the relation of the ${ }^{7} \mathrm{Li}$ production with stellar masses concerning this scenario? We do not have yet a Li production - fine tuning mass relation. This relation will be important for Li galactic evolutionary models. Nevertheless, Drake (1998) has found a relation of $\mathrm{Li}$ depletion with mass among the low-mass giant stars. Giants with $\sim 1 M_{\odot}$ have smaller Li depletion times of the order of $3 \times 10^{3}$ yrs whereas larger depletion times ( $\geq 10^{4} \mathrm{yrs}$ ) appear to be common for giants with $\sim 2 M_{\odot}$. These considerations result for CS expansion velocities of the less than $5 \mathrm{~km} \mathrm{~s}^{-1}$ and mass losses between $10^{-8}-{ }^{-} 10^{-7} M_{\odot} / \mathrm{yr}$.

There are several ways to test the prompt ${ }^{7} \mathrm{Li}$ enrichment - mass loss scenario. Fekel et al. (1996) have found that the star HDE 233517 is not a $\beta$ Pictoris dwarf star as had been considered before (Skinner et al. 1995). On the contrary, this is a distant very Li-rich $\mathrm{K}$ giant. Fekel et al. also showed that the size of CS measured by Skinner et al. (1995) at 10 microns corresponds to what is expected by a Li-strong giant in the prompt ${ }^{7} \mathrm{Li}$ enrichment - mass loss scenario.

Detecting detached (or multiple detached) CS would be a strong indication of the validity of this scenario. Observations by means of $\mathrm{CO}$ lines have beautifully shown the existence of detached and almost perfect spherical CSs and even multiple CSs, among the carbon giant stars (Olofsson et al. 1999; Mauron \& Huggins 1999). To detect detached CSs in RGB stars is, however, much more difficult. The main reason of this difficulty is due to the smaller involved shell masses during the RGB phase. One possibility could be the visualization of the 
dusty CS by FIR images. In the case of HDE 233517, the 10 microns radiation, produced nearby the star, is probably not the most indicated wavelength to detect detached shells. Longer wavelengths measured by ISO could be more convenient. Disappointedly, this satellite has not shown the same clear detached shells in $\mathrm{C}$ giants that have been measured with the CO lines, as far as we know. Other possibilities of exploration of the presence of detached shells can be made in the gas phase of the CS, using the strong Na D lines. Extra absorption features, especially blueward, will indicate the number of shell components, their opacities and their velocities (see Jasniewicz et al. in these proceedings). The appearance of rapid pulses of ${ }^{7} \mathrm{Li}$ enrichment can be detected by observing the simultaneous presence of a very strong ${ }^{7} \mathrm{Li}$ line together with $\mathrm{Be}$ and $\mathrm{B}$ lines and, depending on its position in the $\mathrm{Li}$ cycle, this would be an indication that the star is in the beginning of its ${ }^{7} \mathrm{Li}$ enrichment phase and that the mixing process has not yet destroyed the $\mathrm{Be}$ and $\mathrm{B}$. In the next $\mathrm{Li}$ cycles the $\mathrm{Be}$ and B elements will be already destroyed and only the Li line will be observed (see also Sackmann \& Boothroyd 1999).

\section{External Enrichment Processes}

\subsection{The Nova Mechanism}

In this case, the photosphere of the giant star is enriched with new ${ }^{7} \mathrm{Li}$, produced by a nearby hot nova companion (Gratton \& D' Antona 1989). Even if it is theoretically possible to obtain, under certain conditions, a high production of ${ }^{7} \mathrm{Li}$ by novae, there are no observational evidences of this type of contamination. On the one hand there are no indications of the presence of binary stars among the Li-rich giants due to the absence of radial velocities variations with a precision of $1 \mathrm{~km} \mathrm{~s}^{-1}$ (de Medeiros, Melo \& Mayor 1996). On the other hand, they do not show the presence of a hot companion by means of UV (IUE) spectra (de la Reza \& da Silva 1995).

\subsection{The Planets/Brown Dwarf Engulfing Scenario}

The idea by which giant stars, which had already been depleted by the first dredge-up process, can gain larger Li abundances by accreting planets has been proposed by Alexander (1967). This model has been discussed, introducing the possibility of engulfing also brown dwarf companions, by Gratton \& D 'Antona (1989) and Brown et al. (1989). This scenario has only recently been developed in detail by Siess \& Livio (1999a, 1999b). In the following we will refer only to these last two publications. Depending on the mass of the accreting object, major perturbations of the giant star will occur. The main consequences are the following: a) formation and ejection of the CS producing this way FIR excesses. These authors maintain the same scenario of de la Reza et al. $(1996,1997)$ introducing only the engulfing planets/brown dwarf process at the beginning of the Li cycle. b) increase of the rotational velocity of the giant due to the transfer of angular momentum of the planet. For a typical $1 M_{\odot}$ giant with a radius of $20 R_{\odot}$ the resulting rotational velocity, due to the accretion of a $\sim 5 M_{J u p}$ object, will be of $\sim 5.5 \mathrm{~km} \mathrm{~s}^{-1}$. Larger bodies such as brown dwarfs will produce much faster rotating giants. c) $\mathrm{Li}, \mathrm{Be}$ and $\mathrm{B}$ abundances will increase in the giant, 
but because of their different nuclear fragilities, the effect will be more sensitive for Li. d) the star metallicity will increase. e) Because of the deposition of a new mass, increasing this way the potential energy of the star, an expansion of the stellar envelope will be produced. An accretion of an even larger body can trigger nuclear burning at the base of the convective layer (HBB) in the AGB phase. In general, the engulfing process is more sensitive in the AGB because the stellar envelope is smaller than in the case of the RGB phase. Nevertheless, the accretion of nearby planets as is the case of $51 \mathrm{Peg}$ systems, are expected to be more important in the RGB phase. In any case, no triggering of the CBP is obtained for the low-mass giants. f) Due to the increased rotation, a dynamo activity can be produced resulting in X-ray emission. In principle, at least qualitatively, due to different time scales of all these processes, they are not expected to appear all simultaneously. In a first approach, this scenario appears attractive because something similar (that is a non-simultaneity) is found for a collection of fast rotating giants when compared to their Li abundances, FIR excesses and X-ray emissions (Drake et al. 2000).

Nevertheless, some important major effects which can observationally be tested deserve more attention in this scenario. The most important one is the impossibility of this scenario to explain the presence of very high Li abundances of several RGB stars with abundances larger than the interstellar Li abundance (de la Reza \& da Silva 1995; Pavlenko, Savanov \& Yakovina 1999; Terra 1997). Also, very low rotation velocities $\left(<2 \mathrm{~km} \mathrm{~s}^{-1}\right)$ and very strong $\mathrm{Li}$ abundances $(\log \varepsilon(\mathrm{Li})=3.70)$ as is the case of the giant HD 19745 (de la Reza \& da Silva 1995; Terra 1997; Terra et al. 2000) remain even less explainable. A definite test will be made soon (de la Reza, Cunha, \& Smith in preparation) using the Hubble telescope. This will consist in measuring the boron UV lines. If the engulfing process is possible, we expect the boron (the least fragile among the primordial elements) lines to be larger due to the addition of the boron from the star and that of the planet. This will be especially the case for the limiting high rotating, very Li-rich giant HD $9746\left(v \sin i \sim 8 \mathrm{~km} \mathrm{~s}^{-1}\right)$.

\section{Non- ${ }^{-}$LTE Lithium Abundances Determinations}

There are several physical reasons why Non-LTE (NLTE) is important in the line formation and $\mathrm{Li}$ abundance determinations in Li-strong giants. These are: a) the core of the line appears sometimes saturated and is formed in the very external layers of the photosphere b) the chromospheric radiation affects the ionization of the neutral lithium atom. c) the LTE Li abundance determinations often give different values for different $\mathrm{Li}$ lines.

The importance of the chromosphere comes from the fact that its UV radiation is critical for the neutral $\mathrm{Li}$ atom ionization. In fact, the radiation below $3500 \AA$ producing the ionization from the first excited level $2 \mathrm{~S}$ largely controls the ionization equilibrium of the neutral $\mathrm{Li}$ atom, due to a large photoionization cross-section corresponding to this bound-free transition.

Carlsson et al. (1994) discussed the NLTE Li atom line formation in cool stars generally. NLTE applications to specific cases of Li-strong $\mathrm{K}$ giants were made by de la Reza \& da Silva (1995); Pavlenko, Savanov, \& Yakovina (1999), and Abia, Pavlenko, \& de Laverny (1999). In some cases quite large Li abun- 
dances have been obtained, much larger than the interstellar $\mathrm{Li}$ abundance of $\log \varepsilon(\mathrm{Li}) \sim 3.1$ transforming these giants in important candidates for sources of $\mathrm{Li}$ in the Galaxy. The Li NLTE abundances presented by de la Reza \& da Silva (1995) are, however, somewhat overestimated, because the UV opacity has not directly been taken into account producing this way an extra overionization. New calculations resulting in somewhat reduced abundance values remain, however, larger than the interstellar value and larger than LTE Li abundances. These new values are the result of a self-consisting iterative methodology taking into account the chromospheric effects (Terra 1997; Terra, de la Reza, \& Batalha 2000). In a first step, the chromosphere is fixed by introducing appropriate parametric sources of the UV continuum opacities and by fitting, on the one hand, the observed UV (IUE) continuum and, on the other hand, the observed $\mathrm{H} \alpha$ line profile. This last calculation is made into the iteration scheme, in a separate NLTE calculations of the hydrogen lines. Hereafter, all possible Li lines (the resonance Li I line at $6708 \AA$, and the secondary lines at $6104 \AA$ and $8123 \AA$ ) are calculated in NLTE, using the same Li abundance for all the lines as a main free parameter. The iteration is repeated until a convenient convergence is obtained.

The methodology followed by Pavlenko, Savanov \& Yakovina (1999) is different in the sense that no direct chromospheric effects have been taken into account and NLTE calculations have been realized separately for each line. A satisfying solution was obtained when the $\mathrm{Li}$ abundances were similar for all the lines. A common case of study of these two methods is the case of the $\mathrm{Li} \mathrm{K}$ giant HD 9746, where the resulting values are: $\log \varepsilon(\mathrm{Li})=3.60 \pm 0.3$ in the case of Pavlenko et al. and $\log \varepsilon(\mathrm{Li})=3.80$ in the case of Terra (1997). Considering very high derived NLTE Li abundances, one impressive case is that of the field $\mathrm{K}$ giant PDS 68, which appears to be the richest Li K giant known up to now with a value of $\log \varepsilon(\mathrm{Li})=4.60$ (Terra 1997). This giant star presents an observed secondary $\mathrm{Li}$ l line at $6104 \AA$ with an equivalent width of $280 \mathrm{~m} \AA$ !

\section{Conclusions and Perspectives}

Considering the different scenarios we believe that we can begin to make our choices. The engulfing planets/brown dwarfs scenario have a very strong limitation. At least, as far as it has been developed until now, this scenario is unable to explain the existence of low mass RGB stars with $\mathrm{Li}$ abundances larger than that of the interstellar medium. Naturally it is expected that the interstellar $\mathrm{Li}$ abundance is the one of the engulfed objects in this scenario. Only two solutions appear to solve this problem 1) to accept accreting bodies containing $\mathrm{Li}$ abundances larger by one or two orders of magnitude respect to the interstellar medium. This appears to be quite artificial. 2) the possibility that the engulfing process could trigger the CBP. This, however, appears not to be the case in the actual model. On the other hand, due to the intrinsic necessity of the giant to shallow massive bodies in the RGB stage in order to get a sensitive contamination, this results in an increasing of its rotation velocity. In this way, the existence of very Li-rich low-mass and low rotation giants remains even less explainable in this scenario. In any case a near future test, mentioned before, using the observed boron lines will definitely discard or not this scenario. 
Under these circumstances we prefer the internal scenarios. Nevertheless, in this case, at least for the low-mass giants, the physical mechanism able to form rapidly a circumstellar shell remains to be found. Maybe a natural source of internal energy can be found in the dissipation of a fast rotating core. We must also not forget that a powerful convection can be the source of a CS as has been shown to be the case in Betelgeuse (Lim et al. 1998).

Recent observations are giving more clues to the understanding of the $\mathrm{Li}$ problem. This is the case of the discovery of very Li-strong giants in clusters. Hill \& Pasquini (1999) discovered one very Li-strong $\mathrm{K}$ giant in the old open cluster Berkeley 21. This kind of discoveries is important to better establish the evolutionary age at which the Li cycle appears. Among globular clusters a very Li-strong RGB giant has been detected in M3 (Kraft et al. 1999) and moderately Li-strong ones in NGC 362 (Smith, Shetrone, \& Keane 1999) and in M5 (Carney, Fry \& Gonzalez 1998). For the case of $\mathrm{M} 3$, and using the ${ }^{7} \mathrm{Li}$ prompt enrichment - mass loss scenario, de la Reza et al. (2000) have shown that a large quantity of ${ }^{7} \mathrm{Li}$ can be produced enriching the intracluster matter of this cluster during the whole life of M3. This new Li is probably lost, by ram pressure, into the galactic disk when M3 crosses several times this disk contributing this way to its Li enrichment. They also suggest that for these low-metal giants, the CBP producing a fresh ${ }^{7} \mathrm{Li}$ enrichment, could be related to the nucleosynthesis of $\mathrm{Na}$ and $\mathrm{Al}$ explaining this way the observed star to star variations of these elements.

Is the prompt ${ }^{7} \mathrm{Li}$ - mass loss scenario also into action in the more advanced AGB stage? This remains to be explored. In any case, all the scenario ingredients are present in carbon giants such as a variety of strengths of Li lines including the most extreme Li lines known up to now, as is the case of WZ Cas, T Ara and IY Hya. Also, these giants present very clearly almost perfect spherical detached CS as is the case of TT Cyg (Olofsson et al. 2000). There are also several C giants presenting multiple CSs (Mauron \& Huggins 1999). Another group that deserves special attention are the carbon $\mathrm{J}$ stars, which could be in a peculiar stage of evolution. These stars are characterized by containing moderately strong $\mathrm{Li}$ lines and by being enriched with ${ }^{13} \mathrm{C}$ (see Lorenz-Martins \& Drake and also Abia \& Isern in these proceedings). When $\mathrm{J}$ and cool type $\mathrm{N}$ carbon giants are placed in an IRAS diagram, they are concentrated in a region presenting more excesses in the 60 microns fluxes. If there is a $\mathrm{Li}$ cycle, it is much shorter than that of the $\mathrm{K}$ giants. This situation being compatible with their larger observed CS expansion velocities $\left(\geq 10 \mathrm{~km} \mathrm{~s}^{-1}\right)$.

Up to now there is no a clear relation between ${ }^{13} \mathrm{C}$ and ${ }^{7} \mathrm{Li}$ enrichments. The first results for K giants (da Silva, de la Reza \& Barbuy 1995; Drake 1998, see also Drake et al. in these proceedings) present no correlations indicating that probably enrichments in ${ }^{13} \mathrm{C}$ and ${ }^{7} \mathrm{Li}$ are acting in different time scales. Another group that deserves attention are the Post-AGB giants. Even if the statistics are poor, some of these stars, such as the Frosty Leo nebula present a relatively strong $\mathrm{Li}$ line.

Two important consequences, one related to the internal mixing and the other to episodic mass loss are the following: As more ${ }^{7} \mathrm{Li}$ is created more ${ }^{3} \mathrm{He}$ is destroyed. The cosmological consequences of this, in limiting the interval of abundances of ${ }^{3} \mathrm{He}$ as a "barionmeter" ", are discussed by Sackmann \& Boothroyd (1999). The episodic mass loss for which a specific physical propulsion 
mechanism remains to be found (thermal pulses are proposed for this purpose in the AGB phase) introduces the notion that mass loss could be an essentially variable phenomenon and that this one begin already in the RGB phase. This does not prevent a less strong continuous mass loss from being also present.

One of the advantages of the prompt ${ }^{7} \mathrm{Li}$ enrichment - mass loss scenario consists in bringing a physical picture of the complete path, joining the element production source in the core of stars to the interstellar medium. By considering the Li cycles at the RGB phase and by establishing that similar cycles are also present in the AGB phase, we will dispose of a complete ensemble of observed ${ }^{7} \mathrm{Li}$ sources in order to have a more realistic model to study the evolution of $\mathrm{Li}$ in the Galaxy.

\section{References}

Abia, C., Isern, J. 1997, MNRAS, 289, L11

Abia, C., Pavlenko, Y., \& de Laverny, P. 1999, A\&A, 351, 273

Alexander, J.B. 1967, The Observatory, 87, 238

Brown, J.A., Sneden, C., Lambert, D.L., \& Dutchover, E. 1989, ApJS, 71, 293

Cameron, A.G.W. 1955, ApJ, 121, 144

Cameron, A.G.W. \& Fowler, W.A. 1971, ApJ, 164, 111

Carlsson, M., Rutten, R.J., Bruls, J.H.M.J., \& Shchukina, N.G. 1994, A\&A, 288,860

Carney, B.W., Fry, A.M., \& Gonzalez, G. 1998, AJ, 116, 2984

Castilho, B.V. et al. 1999, A\&A, 345, 249

Charbonnel, C. 1994, A\&A, 282, 811

da Silva, L., de la Reza, R., \& Barbuy, B. 1995, ApJ, 448, L41

de la Reza, R. \& da Silva, L. 1995, ApJ, 439, 917

de la Reza, R., Drake, N.A., \& da Silva, L. 1996, ApJ, 456, L115

de la Reza, R., Drake, N.A., da Silva, L., Torres, C.A.O., \& Martin, E. L. 1997, ApJ, 482, L77

de la Reza, R., da Silva, L., Drake, N.A, \& Terra, M.A. 2000, ApJ, (Letters May)

de Medeiros, J.R. Melo, C.H. F., \& Mayor, M. 1996, A\&A, 309, 465

Drake, N.A. 1998, PhD Thesis, - Observatório Nacional, - Rio de Janeiro

Drake, N.A., de la Reza, R. da Silva, L., \& Lambert, D.L. 2000, submitted to the AJ

Fekel, F.C. 1988, A Decade of UV Astronomy with the IUE Satellite, ESA, SP-281, Vol. 1, p. 331

Fekel, F.C., \& Balachandran, S. 1993, ApJ, 403, 708

Fekel, F.C., Webb, R.A., White, R.J., \& Zuckerman, B. 1996, ApJ, 462, L95

Gratton, R.G., \& D 'Antona, F. 1989, A\&A, 215, 66

Gregório-Hetem, J., Lepine, J.R.D., Quast, G.R., Torres, C.A.O., \& de la Reza, R. 1992, AJ, 103, 549

Gregório-Hetem, J., Castilho, B.V., \& Barbuy, B. 1993, A\&A, 268, L25 
Hill, V., \& Pasquini, L. 1999, A\&A, 348, L21

Kraft, R.P. et al. 1999, ApJ, 518, L53

Lim, J., Carilli, C.L., White, S.M., Beasley, J., \& Marson, R.G. 1998, Nature, 392,575

Mac Kellar, 1940, PASP, 52, 407

Mauron, N., \& Huggins, P.J. 1999, A\&A, 349, 203

Mazzitelli, I., D`'Antona, F., \& Ventura, P. 1999, A\&A, 348, 846

Olofsson, H. et al. 2000, A\&A, 353, 583

Pavlenko, Ya. V., Savanov, I.S., \& Yakovina, L.A. 1999, Astron. Rep., 43, 671

Sackmann, I-J. \& Boothroyd, A.I. 1992, ApJ, 392, L71

Sackmann, I-J. \& Boothroyd, A.I. 1999, ApJ, 510, 217

Siess, L. \& Livio, M. 1999a, MNRAS, 304, 925

Siess, L. \& Livio, M. 1999b, MNRAS, 308, 1133

Skinner, C.J. et al. 1995, ApJ, 444, 861

Smith, V.V., \& Lambert, D.L. 1989, ApJ, 345, 175

Smith, V.V., Shetrone, M.D. \& Keane, M.J. 1999, ApJ, 516, L73

Terra, M.A. 1997, PhD Thesis, Observatório Nacional, - Rio de Janeiro

Terra, M.A., de la Reza, R., \& Batalha, C. 2000, in preparation

Wallerstein, G. \& Sneden, C. 1982, ApJ, 255, 577 